\title{
EFEITO DA RELAÇÃO CÁLCIO E MAGNÉSIO DO CORRETIVO SOBRE MICRONUTRIENTES NA ALFAFA ${ }^{1}$
}

\author{
ADÔNIS MOREIRA², JANICE GUEDES DE CARVALHO, \\ LARISSA ALEXANDRA CARDOSO MORAES ${ }^{4}$ e JOÃO ODEMIR SALVADOR ${ }^{5}$
}

\begin{abstract}
RESUMO - O objetivo deste trabalho foi avaliar o efeito da relação Ca:Mg do corretivo sobre micronutrientes na alfafa, em experimento conduzido em casa de vegetação, em um Latossolo Vermelho-Escuro distrófico. O delineamento experimental utilizado foi, em todos os tratamentos, inteiramente casualizado, com quatro repetições. Foram estudadas cinco relações (1:0, 1:1, 2:1, 3:1, 4:1, na dosagem equivalente a $3.900 \mathrm{~kg} \mathrm{ha}^{-1}$ ), e um tratamento com a dosagem equivalente a $7.800 \mathrm{~kg} \mathrm{ha}^{-1}$ (relação 3:1), em seis cortes, com intervalo de 35 dias. Verificou-se que o dobro da aplicação da relação 3:1 diminui significativamente os teores de $\mathrm{B}, \mathrm{Fe}, \mathrm{Mn}$ e $\mathrm{Zn}$. A aplicação só de $\mathrm{CaCO}_{3}$, como corretivo da acidez, não afeta a absorção de $\mathrm{Cu}$. Os teores dos micronutrientes estudados apresentam níveis considerados adequados, sendo, estes, afetados pelas épocas de corte.
\end{abstract}

Termos para indexação: Medicago sativa, adubação, absorção de nutrientes.

EFFECT OF LIMESTONE CALCIUM AND MAGNESIUM RATIO ON MICRONUTRIENTS IN ALFALFA

\begin{abstract}
This study evaluated the effect of limestone Ca:Mg ratios on micronutrients in alfalfa. A randomized block design was used with five relations of $\mathrm{Ca}: \mathrm{Mg}$ ratios (1:0, 1:1, 2:1, 3:1 and 4:1) at a recommended limestone dosage of $3,900 \mathrm{~kg} \mathrm{ha}^{-1}$. An additional treatment was included at a ratio of $3: 1$ with the dosage of 7,800 $\mathrm{kg} \mathrm{ha}^{-1}$. All treatments had four replicates in a six-cutting number, in 35 days of interval. The variables analyzed were: concentration and quantity of $\mathrm{B}, \mathrm{Cu}, \mathrm{Fe}, \mathrm{Mn}$, and $\mathrm{Zn}$ in dry matter. The decrease of the concentration of $\mathrm{B}, \mathrm{Fe}, \mathrm{Mn}$ and $\mathrm{Zn}$ was obtained in the treatment that used twice the recommended dosage. The antagonic effect between $\mathrm{Ca}$, applied as $\mathrm{CaCO}_{3}$, and $\mathrm{Cu}$ was not observed in the treatments. The concentration of micronutrients varied according to the cutting times.
\end{abstract}

Index terms: Medicago sativa, fertilizer application, nutrient uptake.

${ }^{1}$ Aceito para publicação em 14 de agosto de 2000.

Trabalho financiado pela FAPEMIG.

${ }^{2}$ Eng. Agrôn., Dr., Embrapa-Centro de Pesquisa Agroflorestal da Amazônia Ocidental (CPAA), Caixa Postal 319, CEP 69011-970 Manaus, AM. Bolsista DCR/CNPq. E-mail: adonis@cpaa.embrapa.br

${ }^{3}$ Eng. Agrôn., Dr., Prof. Titular, Dep. de Ciência do Solo, Universidade Federal de Lavras (UFLA), Caixa Postal 37, CEP 37200-000 Lavras, MG. Bolsista do CNPq. E-mail: janice@ufla.br

${ }^{4}$ Eng. Agrôn., M.Sc., Embrapa-CPAA.

E-mail: larissa@cpaa.embrapa.br

${ }^{5}$ Biól., Centro de Energia Nuclear na Agricultura (CENA), Universidade de São Paulo (USP), Caixa Postal 96, CEP 13400-970 Piracicaba, SP. E-mail: salvador@cena.usp.br

\section{INTRODUÇÃO}

As possibilidades de incrementar a produtividade do rebanho leiteiro são determinadas pelo custo e qualidade da alimentação. Dada a qualidade e palatabilidade da alfafa tem sido crescente o interesse pelo seu uso em sistemas intensivos, fazendo com que os animais aumentem seu consumo e, conseqüentemente, a produção. Pela sua versatilidade na utilização (pastejo, feno, silagem e pélete), possibilita ao produtor ajustar a sua produção de acordo com as necessidades de cada animal e as condições climáticas dominantes (Moreira \& Salvador, 1998; Ferreira et al., 1999). 
Diante dessas características, estudos mais aprofundados sobre a adubação da alfafa nas condições de cerrado têm evoluído sistematicamente, e contribuído sobremaneira para o desenvolvimento da eqüinocultura e da pecuária leiteira. Trabalhos recentes mostram o grande potencial desta leguminosa forrageira nessa região (Moreira et al., 1996, 1997a, 1997b).

Em solos de Cerrado, os problemas relacionados com a nutrição de plantas são corrigidos, geralmente, mediante a modificação da disponibilidade de nutrientes com a aplicação de corretivos e fertilizantes, por esses solos apresentarem, na maioria das vezes, baixa fertilidade natural ou toxidez de certos elementos (Fageria \& Morais, 1987).

Sabe-se que com a aplicação de calcário ocorre a elevação do pH e a diminuição na disponibilidade dos micronutrientes, com exceção do Mo (Malavolta, 1980). Outro fator que pode afetar a absorção dos nutrientes é a relação de $\mathrm{Ca}$ e $\mathrm{Mg}$ no solo, os quais podem diminuir ou aumentar a absorção através dos processos de antagonismo, de inibição competitiva ou não, e do sinergismo de alguns elementos, entre eles B, Cu, Fe, Mn e Zn (Malavolta et al., 1997).

O trabalho teve como objetivo avaliar o efeito da relação Ca:Mg do corretivo sobre micronutrientes, na alfafa (Medicago sativa L.).

\section{MATERIAL E MÉTODOS}

O experimento foi conduzido em casa de vegetação do Departamento de Ciência do Solo da Universidade Federal de Lavras (UFLA), região sul de Minas Gerais, localizado nas coordenadas $21^{\circ} 14^{\prime} 6^{\prime \prime}$ de latitude sul e $45^{\circ} 0^{\prime}$ de longitude oeste, a uma altitude média de $900 \mathrm{~m}$, no período de junho de 1992 a maio de 1993.

O solo utilizado foi um Latossolo Vermelho-Escuro distrófico, de cerrado $\left(\mathrm{pH}_{\text {água }}=4,8\right.$; $\mathrm{P}($ Mehlich 1$)=2 \mathrm{mg} \mathrm{dm}^{-3} ; \mathrm{K}($ Mehlich 1$)=40 \mathrm{mg} \mathrm{dm}^{-3}$; $\mathrm{Ca}(\mathrm{KCl})=0,4 \mathrm{cmol}_{\mathrm{c}} \mathrm{kg}^{-1} ; \mathrm{Mg}(\mathrm{KCl})=0,1 \mathrm{cmol}_{\mathrm{c}} \mathrm{kg}^{-1}$; $\mathrm{S}=5,13 \mathrm{mg} \mathrm{dm}^{-3} ; \mathrm{B}$ (água quente) $=0,15 \mathrm{mg} \mathrm{dm}^{-3}$; $\mathrm{Cu}\left(\right.$ Mehlich 1) $=3,2 \mathrm{mg} \mathrm{dm}^{-3} ; \mathrm{Fe}($ Mehlich 1) = $55,4 \mathrm{mg} \mathrm{dm}^{-3} ; \mathrm{Mn}$ (Mehlich 1) = 8,4 $\mathrm{mg} \mathrm{dm}^{-3}$; Zn $\left(\right.$ Mehlich 1) $=0,1 \mathrm{mg} \mathrm{dm}^{-3} ;$ M.O. $=30,8 \mathrm{~g} \mathrm{~kg}^{-1} \mathrm{e}$ $\mathrm{V}=11 \%$ ), coletado no município de Lavras, $\mathrm{MG}$, na camada de $0-25 \mathrm{~cm}$ de profundidade. Após a secagem, o solo foi uniformizado e passado em peneira de malha de $2 \mathrm{~mm}$.

$\mathrm{O}$ delineamento experimental foi o inteiramente casualizado, com seis tratamentos e quatro repetições.
Foram efetuados seis cortes: o primeiro ocorreu três meses após o plantio, e os posteriores, com intervalos de 35 dias. Os tratamentos consistiram de cinco relações Ca:Mg: $1: 0 ; 1: 1 ; 2: 1 ; 3: 1 ; 4: 1$, na dose equivalente a $3.900 \mathrm{~kg} \mathrm{ha}^{-1}$ do corretivo para elevar a saturação por base a $80 \%$. No sexto tratamento foi aplicado o dobro dessa dose na relação 3:1, ou seja, o equivalente a $7.800 \mathrm{~kg} \mathrm{ha}^{-1}$. As relações foram realizadas com $\mathrm{CaCO}_{3}$ e $\mathrm{MgCO}_{3}$.

Dois meses após aplicação dos tratamentos, o solo recebeu adubação básica, nas seguintes doses, em $\mathrm{mg} \mathrm{dm}^{-3}$ : P, 200 (MAP), K, 150 (KCl), S, $50\left(\mathrm{~K}_{2} \mathrm{SO}_{4}\right), \mathrm{B}, 0,5$ $\left(\mathrm{H}_{3} \mathrm{BO}_{3}\right), \mathrm{Co}, 0,01\left(\mathrm{CoCl}_{2} \cdot \mathrm{H}_{2} \mathrm{O}\right), \mathrm{Cu}, 1,5\left(\mathrm{CuSO}_{4} \cdot 5 \mathrm{H}_{2} \mathrm{O}\right)$, $\mathrm{Mo}, 0,1\left(\mathrm{H}_{2} \mathrm{MoO}_{4}\right), \mathrm{Mn}, 3,5\left(\mathrm{MnSO}_{4} \cdot \mathrm{H}_{2} \mathrm{O}\right)$ e $\mathrm{Zn}, 5,0$ $\left(\mathrm{ZnSO}_{4} \cdot 7 \mathrm{H}_{2} \mathrm{O}\right)$, estando essas de acordo com Allen et al. (1976) e Malavolta (1980), para experimentos conduzidos em condições de casa de vegetação. Foi feita a inoculação de Rhizobium meliloti nas sementes. No segundo e quarto corte, foram efetuadas as adubações de manutenção com cloreto de potássio $\left(50 \mathrm{mg} \mathrm{kg}^{-1}\right)$, e no terceiro corte, com micronutrientes $(\mathrm{B}, \mathrm{Cu}, \mathrm{Mn}$ e $\mathrm{Zn}$, nas doses iguais às da instalação do experimento).

Foram semeadas dez sementes escarificadas e infectadas, em vasos com cinco litros de capacidade. Após o desbaste, foram mantidas cinco plantas uniformes. Utilizou-se a cultivar Crioula como planta-teste. Os vasos foram irrigados até que atingissem $70 \%$ do valor total de poros (VTP), e pesados diariamente, a fim de se determinar a quantidade de água a ser reposta. Após cada corte, o material foi pesado e levado a estufa a $65^{\circ} \mathrm{C}$, permanecendo até atingir peso constante. Após a pesagem, o material foi moído em moinho tipo Wiley, e digerido, para obtenção dos extratos. Os teores de $\mathrm{B}, \mathrm{Cu}, \mathrm{Fe}, \mathrm{Mn}$ e $\mathrm{Zn}$ foram determinados conforme os métodos descritos por Malavolta et al. (1997).

Os resultados foram submetidos a análise de variância e teste de comparação de médias dos tratamentos, adotando-se o teste de Tukey a 5\% de probabilidade (PimentelGomes, 1990).

\section{RESULTADOS E DISCUSSÃO}

As relações de Ca:Mg não refletiram significativamente em maiores produções de matéria seca (MS), mas a alfafa foi responsiva com o aumento da aplicação do corretivo (Tabela 1). Tais resultados corroboram os obtidos por Gomes et al. (1995) ao estudarem cinco relações de Ca: $\mathrm{Mg}$ (100:0, 75:25, 50:50, 25:75 e 0:100), e também não obtiveram respostas significativas com a cultivar Crioula no incremento da produção. Com relação ao aumento da quantida- 
de de MS em razão das doses do corretivo, os resultados do presente experimento também concordam com os de Deolindo et al. (1992) e Kornelius \& Ritchey (1992), que nas condições de Curitiba, PR, e Brasília, DF, observaram que esta leguminosa aumentou significativamente a produção com aplicação do dobro da dose recomendada de calcário.

Os teores de B, Fe, Mn e Zn foram afetados negativamente, com aplicação do dobro da dose recomendada (7.800 kg ha-1, relação 3:1), e no caso do Mn e $\mathrm{Zn}$ diferiram estatisticamente dos demais tratamentos (Tabela 1). Com o aumento do pH, em conseqüência do incremento na aplicação do corretivo, pode haver uma insolubilização do $\mathrm{Zn}$ e principalmente do Fe (Malavolta, 1980; Tisdale et al., 1993), este último por ser o solo utilizado naturalmente rico nesse nutriente (Tabela 2).

Com o aumento da dose do corretivo, o Mn foi o elemento mais afetado com relação a sua disponibilidade (Tabela 1). Nas relações 3:1 $\left(3.900 \mathrm{~kg} \mathrm{ha}^{-1} \mathrm{e}\right.$ $7.800 \mathrm{~kg} \mathrm{ha}^{-1}$ ), o teor de Mn sofreu redução de 55,0\%; em seguida, o Zn em 31,4\%; o Cu em 5,9\%; o B em $4,8 \%$; e o Fe em 4,7\%. Spehar (1993), em experimento com 28 cultivares de soja, observou que aplicação do calcário dolomítico, sendo um tratamento com calagem parcial, e outro com a dose recomendada com base nos resultados de análise, os teores foliares de $\mathrm{Cu}, \mathrm{Fe}, \mathrm{Mn}$ e $\mathrm{Zn}$ apresentaram reduções médias de 40,61, 310 e 78\%, respectivamente.

No caso do $\mathrm{Mn}$, o aumento do $\mathrm{pH}$ pode ter inibido a absorção do elemento, dada a diminuição na concentração hidrogeniônica, favorecendo, com isso, a conversão do Mn trocável em formas insolúveis, como o $\mathrm{Mn}^{3+}$ e $\mathrm{Mn}^{4+}$ (Malavolta et al., 1997). Segundo Pendias \& Pendias (1984), o Ca, Mg e Mn apresentam valência, raio iônico e grau de hidratação semelhantes; o aumento da concentração de um pode inibir a absorção do outro.

O menor teor de B no tratamento com $7.800 \mathrm{~kg} \mathrm{ha}^{-1}$ (Tabela 1) ocorreu talvez como conseqüência da formação de compostos pouco solúveis, em decorrência do aumento da quantidade de matéria orgânica no solo (Malavolta, 1980). A alfafa, por ter produzido, nesse tratamento, cerca de $32 \%$ mais MS que os outros, deve ter acarretado um maior volume de raízes, e, com a morte destas, aumentou a quantidade de matéria orgânica nos vasos. Outro motivo, segundo Su et al. (1994), é que com a elevação do pH e da quantidade de $\mathrm{CaCO}_{3}$ adicionada no solo, a adsorção de B é significativamente aumentada, diminuindo, com isso, a sua disponibilidade para a planta.

$\mathrm{O}$ aumento da concentração de $\mathrm{CaCO}_{3}$ como corretivo da acidez não diminuiu significativamente os teores de Cu na matéria seca total (Tabela 1). Segundo Malavolta (1980), Marschner (1995) e Malavolta et al. (1997), no antagonismo, a presença de um elemento diminui a absorção do outro, cuja toxidez pode ser evitada: o $\mathrm{Ca}^{2+}$ impede a absorção exagerada de $\mathrm{Cu}^{2+}$. Tal fato, no presente trabalho, não ocorreu.

Os dados da Tabela 3 indicam que o conteúdo dos micronutrientes foram significativamente afetados pelo aumento da produção de matéria seca (Tabe-

TABELA 1. Produção de matéria seca e teores de B, Cu, Fe, Mn e Zn na alfafa, em razão dos tratamentos (média dos seis cortes e das quatro repetições) ${ }^{1}$.

\begin{tabular}{|c|c|c|c|c|c|c|}
\hline $\begin{array}{c}\text { Tratamento } \\
\mathrm{Ca}: \mathrm{Mg}\end{array}$ & $\begin{array}{c}\text { MS } \\
\text { (g/vaso) }\end{array}$ & B & $\mathrm{Cu}$ & $\begin{array}{c}\mathrm{Fe} \\
\left(\mathrm{mg} \mathrm{kg}^{-1}\right)\end{array}$ & $\mathrm{Mn}$ & $\mathrm{Zn}$ \\
\hline $1: 0$ & $10,3 b$ & $68,67 \mathrm{a}$ & $10,44 \mathrm{a}$ & $214,53 b$ & $70,04 b$ & $68,14 c$ \\
\hline $1: 1$ & $10,2 b$ & $65,54 \mathrm{ab}$ & $11,88 \mathrm{a}$ & $257,44 a$ & $75,04 b$ & $97,59 a$ \\
\hline $2: 1$ & $10,2 b$ & $63,18 \mathrm{ab}$ & $10,69 a$ & $216,35 b$ & $72,94 b$ & $91,33 \mathrm{ab}$ \\
\hline $3: 1$ & $11,2 b$ & $59,98 \mathrm{ab}$ & $12,65 a$ & $199,01 b$ & $82,14 a$ & $88,14 b$ \\
\hline $4: 1$ & $9,6 b$ & $60,07 \mathrm{ab}$ & $12,57 \mathrm{a}$ & $197,84 b$ & $69,21 b$ & $89,55 \mathrm{ab}$ \\
\hline $3: 1^{2}$ & $15,2 \mathrm{a}$ & $57,10 b$ & $11,90 \mathrm{a}$ & $189,64 b$ & $36,99 \mathrm{c}$ & $60,44 c$ \\
\hline Média & 11,1 & 62,42 & 11,69 & 212,48 & 67,73 & 82,53 \\
\hline D.M.S. & 1,8 & 8,70 & 2,23 & 27,31 & 6,85 & 8,65 \\
\hline
\end{tabular}

${ }^{1}$ Médias seguidas por letras distintas, em cada coluna, diferem entre si a 5\% de probabilidade pelo teste de Tukey.

2 Dobro da quantidade aplicada na relação $3: 1$ (dose equivalente a $7.800 \mathrm{~kg} \mathrm{ha}^{-1}$ ). 
la 1). Assim, com aplicação de $7.800 \mathrm{~kg} \mathrm{ha}^{-1}$ do corretivo, exceto o conteúdo de $\mathrm{Mn}$ e $\mathrm{Zn}$, os maiores teores dos demais micronutrientes foram encontrados nesse tratamento.

Com relação às épocas de corte, observa-se a influência da absorção de micronutrientes pela alfafa (Tabela 4). Em todas as relações de Ca:Mg, o teor e conteúdo de $\mathrm{Fe}$ e $\mathrm{Zn}$ tiveram os maiores valores na última amostragem (sexto corte). Tal resultado provavelmente ocorreu em virtude dos efeitos de concentração descritos por Malavolta (1980) e Marschner (1995).
Os teores de $\mathrm{Cu}$ e $\mathrm{Mn}$ foram maiores no quinto corte, enquanto o B seguiu a mesma tendência do conteúdo presente na matéria seca. De acordo com os dados, não foram encontradas justificativas plausíveis para essas variações nos teores e conteúdo dos micronutrientes na matéria seca da parte aérea. Em decorrência dos cortes realizados, esperava-se que todos os nutrientes analisados - exceto o Fe, que não fez parte do programa de adubação - apresentassem comportamentos semelhantes, visto que esses nutrientes foram aplicados na mesma época, ou seja, uma adubação de plantio e outra de manutenção (após

TABELA 2. Resultado das análises químicas de B (água quente), $\mathrm{Cu}, \mathrm{Fe}, \mathrm{Mn}$ e Zn (extrator Mehlich 1) no solo antes do plantio e depois do último corte (média das quatro repetições) ${ }^{\mathbf{1}}$.

\begin{tabular}{|c|c|c|c|c|c|c|c|}
\hline $\begin{array}{c}\text { Tratamento } \\
\mathrm{Ca}: \mathrm{Mg}\end{array}$ & $\begin{array}{c}\mathrm{pH} \\
\left(\mathrm{em} \mathrm{H} \mathrm{H}_{2} \mathrm{O}\right)\end{array}$ & B & $\mathrm{Cu}$ & $\begin{array}{l}\mathrm{Fe} \\
\mathrm{mg} \mathrm{dn}\end{array}$ & $\mathrm{Mn}$ & $\mathrm{Zn}$ & $\begin{array}{l}\mathrm{V} \\
(\%)\end{array}$ \\
\hline & \multicolumn{7}{|c|}{ Antes do plantio } \\
\hline $1: 0$ & 6,4 & 0,41 & 3,1 & 62,9 & 9,9 & 19,70 & 64,3 \\
\hline $1: 1$ & 6,3 & 0,40 & 2,9 & 79,4 & 18,0 & 11,30 & 59,3 \\
\hline $2: 1$ & 6,0 & 0,74 & 3,0 & 81,8 & 13,0 & 18,35 & 45,0 \\
\hline $3: 1$ & 6,3 & 0,70 & 3,2 & 72,6 & 17,1 & 11,25 & 61,5 \\
\hline $4: 1$ & 6,1 & 0,88 & 3,0 & 61,3 & 13,0 & 20,05 & 59,3 \\
\hline \multirow[t]{2}{*}{$3: 1$} & 6,6 & 0,75 & 2,8 & 70,4 & 14,8 & 15,00 & 65,8 \\
\hline & \multicolumn{7}{|c|}{ Após o último corte } \\
\hline 1:0 & 4,8 & 0,20 & 1,5 & 72,3 & 9,8 & 17,46 & 31,8 \\
\hline $1: 1$ & 4,9 & 0,14 & 1,1 & 75,9 & 11,1 & 12,81 & 33,0 \\
\hline $2: 1$ & 4,8 & 0,35 & 1,7 & 61,9 & 11,2 & 13,54 & 31,8 \\
\hline $3: 1$ & 4,7 & 0,54 & 1,0 & 85,4 & 11,4 & 14,26 & 30,0 \\
\hline $4: 1$ & 4,9 & 0,45 & 1,2 & 82,2 & 10,8 & 14,99 & 32,3 \\
\hline $3: 1$ & 5,8 & 0,36 & 1,0 & 87,1 & 13,1 & 17,40 & 59,3 \\
\hline
\end{tabular}

${ }^{1}$ Nos cinco primeiros tratamentos foi aplicada uma dose equivalente a $3.900 \mathrm{~kg} \mathrm{ha}^{-1} \mathrm{e}$ no último foi aplicada uma dose equivalente a $7.800 \mathrm{~kg}^{-1}$.

TABELA 3. Conteúdo de B, Cu, Fe, Mn e Zn na alfafa, em razão dos tratamentos (média dos seis cortes e das quatro repetições) ${ }^{1}$.

\begin{tabular}{|c|c|c|c|c|c|}
\hline Tratamento & B & $\mathrm{Cu}$ & $\mathrm{Fe}$ & $\mathrm{Mn}$ & $\mathrm{Zn}$ \\
\hline $\mathrm{Ca}: \mathrm{Mg}$ & ------------ & --- & $-(\mu \mathrm{g} / \mathrm{vaso})$ & & --- \\
\hline $1: 0$ & $707,30 \mathrm{ab}$ & $107,53 \mathrm{c}$ & $2.209,66 c$ & $721,41 b c$ & $701,84 \mathrm{c}$ \\
\hline $1: 1$ & $668,51 b c$ & $121,18 \mathrm{c}$ & $2.625,89 \mathrm{ab}$ & $765,41 b$ & $995,42 \mathrm{a}$ \\
\hline $2: 1$ & $644,44 b c$ & $119,73 \mathrm{c}$ & $2.206,77 \mathrm{c}$ & $743,93 b c$ & $931,57 \mathrm{a}$ \\
\hline $3: 1$ & $651,28 b c$ & $141,68 b$ & $2.228,91 b c$ & $919,97 a$ & $987,17 \mathrm{ab}$ \\
\hline $4: 1$ & $573,22 \mathrm{c}$ & $120,67 \mathrm{c}$ & $1.899,26 \mathrm{c}$ & $664,42 \mathrm{c}$ & $859,68 b$ \\
\hline $3: 1^{2}$ & $867,92 \mathrm{a}$ & $180,88 \mathrm{a}$ & $2.882,53 \mathrm{a}$ & $562,25 d$ & $918,68 \mathrm{a}$ \\
\hline D.M.S. & 123,20 & 19,84 & 403,99 & 99,77 & 128,89 \\
\hline
\end{tabular}

1 Médias seguidas por letras distintas, em cada coluna, diferem entre si a 5\% de probabilidade pelo teste de Tukey.

2 Dobro da quantidade aplicada na relação 3:1 (dose equivalente a $7.800 \mathrm{~kg} \mathrm{ha}^{-1}$ ). 
TABELA 4. Teores de B, Cu, Fe, Mn e Zn nos seis cortes realizados (média de todos os tratamentos e das quatro repetições) ${ }^{1}$.

\begin{tabular}{lccccc}
\hline Corte & $\mathrm{B}$ & $\mathrm{Cu}$ & $\mathrm{Fe}$ & $\mathrm{Mn}$ & $\mathrm{Zn}$ \\
& - & $-\mathrm{c}$ & \\
\hline $1^{\mathrm{o}}$ & $72,70 \mathrm{a}$ & $11,29 \mathrm{~b}$ & $154,02 \mathrm{c}$ & $53,29 \mathrm{e}$ & $25,66 \mathrm{f}$ \\
$2^{\mathrm{o}}$ & $58,47 \mathrm{~b}$ & $12,35 \mathrm{ab}$ & $145,39 \mathrm{c}$ & $63,45 \mathrm{~cd}$ & $50,15 \mathrm{e}$ \\
$3^{\mathrm{o}}$ & $57,05 \mathrm{~b}$ & $11,75 \mathrm{~b}$ & $150,19 \mathrm{c}$ & $69,95 \mathrm{bc}$ & $65,94 \mathrm{~d}$ \\
$4^{\mathrm{o}}$ & $60,10 \mathrm{~b}$ & $11,99 \mathrm{~b}$ & $142,17 \mathrm{c}$ & $74,23 \mathrm{~b}$ & $104,80 \mathrm{c}$ \\
$5^{\mathrm{o}}$ & $58,05 \mathrm{~b}$ & $13,79 \mathrm{a}$ & $235,25 \mathrm{~b}$ & $86,15 \mathrm{a}$ & $116,26 \mathrm{~b}$ \\
$6^{\mathrm{o}}$ & $54,08 \mathrm{~b}$ & $8,94 \mathrm{c}$ & $447,79 \mathrm{a}$ & $59,27 \mathrm{de}$ & $132,35 \mathrm{a}$ \\
\hline Média & 60,07 & 11,68 & 212,47 & 67,72 & 82,53 \\
\hline D.M.S. & 8,17 & 1,67 & 27,31 & 6,85 & 8,64 \\
\hline
\end{tabular}

${ }^{1}$ Médias seguidas por letras distintas, em cada coluna, diferem entre si a $5 \%$ de probabilidade pelo teste de Tukey.

o terceiro corte). Mesmo assim, esses resultados corroboram os obtidos por Moreira (1997), em trabalho realizado em casa de vegetação com a cultivar Florida 77, nos quais foram também realizados seis cortes, porém com intervalo de 30 dias.

As médias dos teores de $\mathrm{B}, \mathrm{Cu}, \mathrm{Fe}, \mathrm{Mn}$ e $\mathrm{Zn}$ encontradas na matéria seca da parte aérea da alfafa foram adequadas, ficando próximas da faixa dos teores tidos como adequados (B, 20 a 80; Cu, 5 a 30; Fe, 60 a 200; Mn, 25 a 100; Zn, 20 a 70 mg kg-1) por Rhykerd \& Overdahl (1972), Pinkerton et al. (1997) e Moreira et al. (1997b).

\section{CONCLUSÕES}

1. O aumento da dose do corretivo diminui significativamente os teores de $\mathrm{B}, \mathrm{Fe}, \mathrm{Mn}$ e $\mathrm{Zn}$ na matéria seca.

2. A elevação da concentração de Ca no corretivo até a dose aplicada não afeta a absorção de $\mathrm{Cu}$ pela alfafa.

3. O teor e o conteúdo dos micronutrientes são afetados pelas sequiências de corte.

4. O aumento da dose de calcário e a manutenção dos micronutrientes em níveis adequados eleva significativamente a produção de matéria seca da alfafa.

\section{REFERÊNCIAS}

ALLEN, S.E.; TERMAN, G.L.; CLEMENTS, L.B. Greenhouse techniques for soil-plant-fertilizer research. Muscle Shoals : National Fertilizer Development Center, 1976. 55p.

DEOLINDO, J.V.P.; RONCATO, L.D.B.; WATANABE, P.; USHIWATA, C.T.; PREVEDELLO, B.M.S.; PEREIRA, E. Efeito de cinco níveis de calagem na cultura da alfafa (Medicago sativa $\mathrm{L}$.) cultivada em cambissolo, sob condições de casa de vegetação. In: REUNIÃO BRASILEIRA DE FERTILIDADE DO SOLO E NUTRIÇÃO DE PLANTAS, 20., Piracicaba, 1992. Anais. Piracicaba : Sociedade Brasileira de Ciência do Solo/ESALQ, 1992.p.436-437.

FAGERIA, N.K.; MORAIS, O.P. Avaliação de cultivares de arroz na utilização de cálcio e magnésio em solo de cerrado. Pesquisa Agropecuária Brasileira, Brasília, v.22, n.7, p.667-672, jul. 1987.

FERREIRA, R.P.; BOTREL, M.A.; PEREIRA, A.V.; CRUZ, C.D. Avaliação de cultivares de alfafa e estimativas de repetibilidade de caracteres forrageiros. Pesquisa Agropecuária Brasileira, Brasília, v.34, n.6, p.995-1002, jun. 1999.

GOMES, F.T.; BORGES, A.C.; BORGES, J.C.L.; FONTES; P.C.R. Produção de matéria seca e absorção de cálcio e magnésio na alfafa, em resposta à calagem, com diferentes relações cálcio:magnésio. In: $\mathrm{CON}$ GRESSO BRASILEIRO DE CIÊNCIA DO SOLO, 25., Viçosa, 1995. Anais. Viçosa : Sociedade Brasileira de Ciência do Solo/UFV, 1995. p.1120-1121.

KORNELIUS, E.; RITCHEY, K.D. Comportamento da alfafa em diferentes níveis de acidez do solo. Pesquisa Agropecuária Brasileira, Brasília, v.27, n.2, p.241-246, fev. 1992.

MALAVOLTA, E. Elementos de nutrição mineral de plantas. Piracicaba: Agronômica Ceres, 1980.251p.

MALAVOLTA, E.; VITTI, G.C.; OLIVEIRA, S.A. Avaliação do estado nutricional das plantas: princípios e aplicações. Piracicaba : Associação Brasileira para Pesquisa da Potassa e do Fosfato, 1997. 319p.

MARSCHNER, H. Mineral nutrition of higher plants. London : Academic, 1995. 889p.

MOREIRA, A. Efeito de fontes e doses de fósforo na alfafa (Medicago sativa L.) e Centrosema (Centrosema pubescens Benth) e avaliação de extratores. Piracicaba : ESALQ, 1997. 107p. Dissertação de Mestrado.

MOREIRA, A.; CARVALHO, J.G.; EVANGELISTA, A.R. Efeito de doses de enxofre na produção e composi-

Pesq. agropec. bras., Brasília, v.35, n.10, p.2051-2056, out. 2000 
ção mineral da alfafa. Pesquisa Agropecuária Brasileira, Brasília, v.32, n.5, p.533-538, maio 1997a.

MOREIRA, A.; EVANGELISTA, A.R.; CARVALHO, J.G.; SALVADOR, J.O. Efeito de fontes e doses de enxofre nos teores e conteúdo de micronutrientes na alfafa e no trevo branco. Boletim da Indústria Animal, Nova Odessa, v.22, n.2, p.55-60, 1997b.

MOREIRA, A.; EVANGELISTA, A.R.; RODRIGUES G.H.S. Avaliação de cultivares de alfafa na região de Lavras, Minas Gerais. Pesquisa Agropecuária Brasileira, Brasília, v.31, n.6, p.707-711, jun. 1996.

MOREIRA. A.; SALVADOR, J.O. Medicago sativa L. NOTESALQ, Piracicaba, v.4, n.23, p.8, 1998.

PENDIAS, A.K.; PENDIAS, H. Trace elements in soils and plants. Boca Raton : CRC, 1984. 315p.

PIMENTEL-GOMES, F. Curso de Estatística Experimental. Piracicaba : Nobel, 1990. 468p.
PINKERTON, A.; SMITH, F.W.; LEWIS, D.C. Pasture species. In: REUTER, D.J.; ROBINSON, J.B. (Ed.) Plant analysis: an interpretation manual. Melbourne : CSIRO, 1997. p.285-343.

RHYKERD, C.L.; OVERDAHL, C.J. Nutrition and fertilizer use. In: HANSON, C.H. (Ed.). Alfalfa science and technology. Madison: American Society of Agronomy, 1972. p.533-569.

SPEHAR, C.R. Composição mineral da soja cultivada em solo sob cerrado com dois níveis de calagem. Pesquisa Agropecuária Brasileira, Brasília, v.28, n.5, p.645-648, maio 1993.

SU, C.; EVANS, L.J.; BATES, T.E.; SPIERS, G.A. Extractable soil boron and alfalfa uptake: calcium carbonate effects on acid soil. Soil Science Society of America. Journal, Madison, v.54, n.5, p.14451450, 1994.

TISDALE, S.L.; NELSON, W.L.; BEATON, J.D.; HAVLIN, J.L. Soil fertility and fertilizer. New York : MacMillan, 1993. 1634p. 